\title{
Editorial
}

\section{Advanced Video Technologies and Applications for H.264/AVC and Beyond}

\author{
Jar-Ferr (Kevin) Yang, ${ }^{1}$ Hsueh-Ming Hang, ${ }^{2}$ Eckehard Steinbach, ${ }^{3}$ and Ming-Ting Sun ${ }^{4}$ \\ ${ }^{1}$ Department of Electrical Engineering, National Cheng Kung University, Tainan 701, Taiwan \\ ${ }^{2}$ Department of Electronic Engineering, National Chao Tung University, Hsinchu 300, Taiwan \\ ${ }^{3}$ Institute of Communication Networks, Munich University of Technology, 80290 Munich, Germany \\ ${ }^{4}$ Department of Electrical Engineering, University of Washington, Seattle, WA 98195, USA
}

Received 3 August 2006; Accepted 3 August 2006

Copyright (c) 2006 Jar-Ferr (Kevin) Yang et al. This is an open access article distributed under the Creative Commons Attribution License, which permits unrestricted use, distribution, and reproduction in any medium, provided the original work is properly cited.

The recently developed video coding standard, H.264/AVC, significantly outperforms previous standards in terms of coding performance at reasonable implementation complexity. Several application systems, such as high-definition DVD and digital video broadcasting for handheld devices and high-definition television systems, have adopted H.264 or its modified versions as the video coding standard. In addition, the extensions of H.264/AVC to scalable and multiview video coding applications are nearly finalized. Many video services, especially bandwidth-limited wireless video, will benefit from the H.264 coder due to its outstanding features.

The use of variable block sizes for intra- and interprediction in combination with different intra-prediction modes and motion compensation using multiple reference frames is one of the main reasons for the improved coding efficiency in H.264/AVC. Together with many other new features, the encoder can select between multitudes of different coding modes.

The determination of the optimal coding mode under the joint rate and distortion consideration, which is called the rate-distortion optimization (RDO), introduces a huge amount of memory access and computational complexity for testing all possible modes in video encoders. Hence, a reduction of the complexity of motion estimation and mode selection in an H.264/AVC encoder becomes an important task for real-time applications. In selecting the quantization parameters at the frame and the block levels, the goal is to design a rate-control method that maximizes the video quality with a constrained bandwidth. Regardless of the superior coding efficiency of the H.264/AVC standard, there still exist many video coding standards. For example, MPEG-2 and H.263 have been adopted by the current television and video telephony systems, respectively. Therefore, an effective transcoding method, which can effectively convert the existing nonH.264 bitstreams to H.264 conforming bitstreams, while maintaining the excellent rate-distortion performance, will greatly smooth the transition in the migration to H.264/AVC.

Compressed video is typically the most demanding component in modern multimedia services. The statistical analysis on H.264/AVC bitstreams will help to precisely characterize the traffic in video communication. Furthermore, the accurate prediction of dynamic bandwidth allocation of video encoders in network utilization is also important to achieve the best quality of service (QoS). Over wireless and Internet communications, transmission rate variations and transmission-error/packet-loss are inevitable during video streaming. To provide seamless services, the switching capabilities provided by the H.264/AVC standard should be intelligently used to adapt to changing channel characteristics. It has been noted that the more the video is compressed, the more the decoder suffers from error propagation and picture degradation in the case of data loss. Identifying the critical bits of an H.264/AVC bitstream and adding in various degrees of protection can provide a more robust video transmission. Various techniques such as unequal error protection, prioritized transmission, and proper slice insertion in the H.264/AVC stream can further enhance its error resilient features.

This EURASIP JASP special issue, entitled "Advanced video technologies and applications for H.264/AVC and beyond," presents eleven recent research papers related to H.264/AVC. They cover a wide spectrum including the following important topics: adaptive backward motion 
prediction, fast motion estimation and mode selection, fast rate-distortion optimization (RDO), rate control, H.263 to H.264 transcoding, long video tracing, switched streaming, and error protection. These papers can generally be grouped into two main categories based on their contributions: (1) H.264/AVC fast parameter selection and rate-control, and (2) H.264/AVC video bitstream modeling and error protection techniques for video transmission. A summary of the papers in these two categories is given below.

The first five papers address the issues related to fast or optimal parameter selection and rate-control techniques that improve either coding performance or coding speed.

The first paper, "Least-square prediction for backward adaptive video coding," by Li discusses a least-square prediction technique using the duality between the edge contour in images and the motion trajectory in video to achieve a better prediction than the $4 \times 4$, full-search, quarter-pel block matching algorithm without transmitting any overhead. This better prediction will improve the coding efficiency.

The paper, "Fast motion estimation and intermode selection for H.264," by Choi et al. presents a multi-frame/multiresolution motion estimation method using the Hexagon search. For fast inter-mode selection, a bottom-up merge strategy is suggested.

In "Scalable fast rate-distortion optimization for H.264/AVC," Pan et al. design a scalable fast RDO algorithm to effectively choose the best coding mode by initially searching the most probable modes.

The paper, "Rate control for H.264 with two-step quantization parameter determination but single-pass encoding," by Yang et al. proposes an efficient rate-control strategy for H.264, which maximizes the video quality by determining the quantization parameter $(\mathrm{QP})$ for each macroblock. With a preanalysis coarse QP, the refinement of the QP is further enhanced by using the information of motion-compensated residues.

By adopting motion estimation and rate control mechanism, the paper "Efficient video transcoding from H.263 to H.264/AVC standard with enhanced rate control," by Nguyen and Tan devises an H.263 to H.264 transcoding system based on a motion vector reestimation scheme and a fast intra-prediction mode selection. An enhanced rate-control method based on a quadratic model for selecting quantization parameters is also suggested.

The next six papers discuss video bitstream modeling and error protection techniques for effectively transmitting the H.264/AVC bitstreams.

In "H.264/AVC video compressed traces: multifractal and fractal analysis," Reljin et al. examine the H.264/AVC video by fractal and multifractal spectra, which can precisely characterize both local and global features such that a more accurate modeling of the compressed video traffic can be achieved.

Dealing with the bandwidth variation issue, the paper, "Optimized H.264-based bit stream switching for mobile video streaming," by Stockhammer et al. exploits the H.264/AVC SP/SI pictures to optimize the encoders by introducing a framework for dynamic switching and frame scheduling. The achievable performance gains over the constant bit-rate encoding are demonstrated for wireless video streaming over enhanced GPRS.

Zhang and Zeng in "Seamless bit-stream switching in multirate-based video streaming systems" propose an efficient switching method by using an independent or a joint processing in the wavelet domain and an SPIHT coding scheme to achieve an improved coding quality on the H.264/AVC SP/SI pictures.

The paper, "H.264 layered coded video over wireless networks: channel coding and modulation constraints," by Ghandi et al. presents the prioritized transmission of H.264 layered coded video over wireless channels by using prioritized forward error correction coding or hierarchical quadrature amplitude modulation to achieve the layered transmission of data-partitioned and SNR-scalable coded video.

In "Robust transmission of H.264/AVC streams using adaptive group slicing and unequal error protection," Thomos et al. present an error resilient scheme for transmission of H.264/AVC video streams over lossy packet networks using Reed-Solomon codes, adaptive classification of macroblocks, and channel rate allocation.

By using an error-resilient unequal error protection method, the paper, "Error-resilient unequal error protection of fine granularity scalable video bitstreams," by Cai et al. proposes a packet loss protection method for streaming the fine granularity scalable video to guarantee the successful decoding of all received bits resulting in strong errorresilience and high robustness video transmission.

The guest editors would like to thank all the authors for their contributions. We would also like to express our deep appreciation to the reviewers for their conscientious efforts in evaluating all the submitted manuscripts and improving readability of the accepted papers. We hope that this special issue will inspire further research work on improving the coding performance of H.264/AVC coders as well as all the practical issues related to the transmission of H.264/AVC coded streams.
Jar-Ferr (Kevin) Yang
Hsueh-Ming Hang
Eckehard Steinbach
Ming-Ting Sun 
Jar-Ferr (Kevin) Yang received the B.S. degree from the Chung-Yuan Christian University, Taiwan, in 1977, the M.S. degree from the National Taiwan University, Taiwan, in 1979, and the Ph.D. degree from the University of Minnesota, Minneapolis, USA, in 1988, all in electrical engineering. He was an instructor in the Chinese Naval Engineering School for his Navy ROTC service in 1979-1980. He, as an Assistant Re-

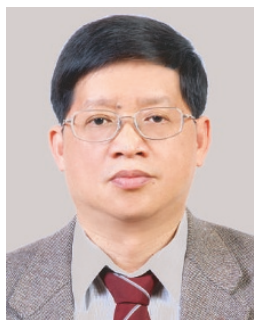
searcher, worked in the Data Transmission and Network Design Research Group, Telecommunication Laboratories, during 19811984. From 1984 to 1988, he received the Government Study Abroad Scholarship that supported his advanced study in the University of Minnesota. In 1988, he jointed the National Cheng Kung University and promoted to the Full Professor in 1994. In 2002, he was a Visiting Scholar at the Department of Electrical Engineering, University of Washington in Seattle, USA. Currently, he is the Director of Graduate Institute of Computer and Communication Engineering, the Director of the Electrical and Information Technology Center, and a Distinguished Professor. During 2004-2005, he is one of speakers in the Distinguished Lecturer Program selected by the IEEE Circuits and Systems Society. He is an Associate Editor of EURASIP Journal of Applied Signal Processing. He is an Associate Editor of the IEEE Circuits and Devices Magazine. He has published over 74 journal and 100 conference papers.

Hsueh-Ming Hang received the B.S. and M.S. degrees from National Chiao Tung University, Hsinchu, Taiwan, in 1978 and 1980, respectively, and the Ph.D. degree in electrical engineering from Rensselaer Polytechnic Institute, Troy, NY, in 1984. From 1984 to 1991, he was with AT\&T Bell Laboratories, Holmdel, NJ. He then joined the Electronics Engineering Department of $\mathrm{Na}-$ tional Chiao Tung University, Hsinchu, Tai-

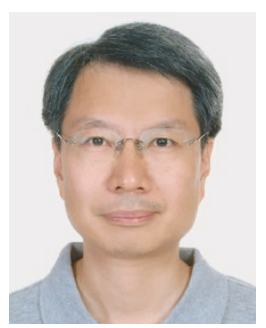
wan, in December 1991. He has been involved in the international video standards since 1984 . His current research interests include digital video compression, image/signal processing algorithm and architecture, and multimedia communication systems. He holds 10 patents and has published over 150 technical papers related to image compression, signal processing, and video codec architecture. He was a coeditor of Optical Engineering special issues on Visual Communications and Image Processing in July 1991 and July 1993, an Associate Editor of the IEEE Transactions on Image Processing (1992-1994) and the IEEE Transactions on Circuits and Systems for Video Technology (1997-1999), and an Area Editor of Journal of Visual Communication and Image Representation, Academic Press (1996-1998). He is a coeditor and contributor of Handbook of Visual Communications published by Academic Press in 1995. He is a recipient of the IEEE Third Millennium Medal and the IEEE ISCE Outstanding Service Award. He is a Fellow of IEEE and a Member of Sigma Xi.

Eckehard Steinbach studied electrical engineering at the University of Karlsruhe, Karlsruhe, Germany, the University of Essex, Colchester, UK, and ESIEE, Paris, France. He received the Engineering Doctorate from the University of ErlangenNuremberg, Germany, in 1999. From 1994 to 2000, he was a Member of the Research Staff of the Image Communication Group

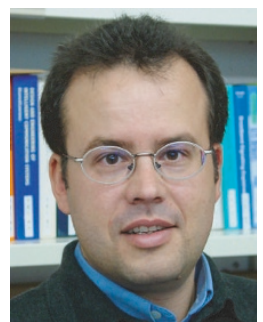

at the University of Erlangen-Nuremberg. From February 2000 to December 2001, he was a Postdoctoral Fellow with the Information Systems Lab at Stanford University. In February 2002, he joined the Department of Electrical Engineering and Information Technology of Technische Universität München, Munich, Germany, as a Professor of media technology. His current research interests are in the area of networked multimedia systems. He served as a Conference Cochair of "SPIE Visual Communications and Image Processing (VCIP)" in San Jose, California, in 2001, and "Vision, Modeling and Visualization 2003 (VMV)" in Munich, in November 2003. He has been a Guest Editor of the Special Issue on Multimedia over IP and Wireless Networks of the EURASIP Journal on Applied Signal Processing in 2004. During 2006-2007 he serves as an Associate Editor for IEEE Transactions on Circuits and Systems for Video Technology (CSVT). In March 2005 he has been appointed as a Guest Professor at the Chinese-German Hochschulkolleg (CDHK) at Tongji University in Shanghai.

Ming-Ting Sun received the B.S. degree from National Taiwan University in 1976, the M.S. degree from University of Texas at Arlington in 1981, and the Ph.D. degree from University of California, Los Angeles in 1985, all in electrical engineering. He joined the University of Washington in August 1996 where he is now a Professor. Previously, he was the Director of the Video Signal Processing Research Group at Bell-

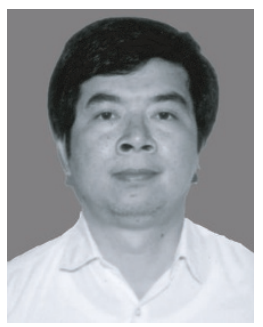
core. He holds 11 patents and has published over 180 technical papers, including 13 book chapters in the area of video and multimedia technologies. He coedited a book Compressed Video over Networks. He was the Editor-in-Chief of the IEEE Transactions on Multimedia (TMM) and a Distinguished Lecturer of the Circuits and Systems Society from 2000 to 2001 . He received an IEEE CASS Golden Jubilee Medal in 2000, and was the General Cochair of the Visual Communications and Image Processing 2000 Conference. He was the Editor-in-Chief of the IEEE Transactions on Circuits and Systems for Video Technology (TCSVT) from 1995 to 1997. He received the TCSVT Best Paper Award in 1993. From 1988 to 1991, he was the Chairman of the IEEE CAS Standards Committee and established the IEEE Inverse Discrete Cosine Transform Standard. He received an Award of Excellence from Bellcore for his work on the digital subscriber line in 1987. 